\title{
Hepatocellular carcinoma and antidepressants: a nationwide population-based study
}

\author{
Vincent Chin-Hung Chen ${ }^{1,2}$, Chiao-Fan Lin ${ }^{2,3}$, Yi-Hsuan Hsieh ${ }^{2,3}$, Hsin-Yi Liang ${ }^{2,3}$, \\ Kuo-You Huang ${ }^{4}$, Wei-Che Chiu ${ }^{5,6, *}$, Yena Lee ${ }^{7}$, Roger S. McIntyre ${ }^{7,8}$ and Hsiang- \\ Lin Chan ${ }^{2,3}$ \\ 1 Department of Psychiatry, Chiayi Chang Gung Memorial Hospital, Chiayi, Taiwan \\ 2 Department of Psychiatry, Chang Gung University, Taoyuan, Taiwan \\ ${ }^{3}$ Department of Child Psychiatry, Linkou Chang Gung Memorial Hospital, Taoyuan, Taiwan \\ ${ }^{4}$ Department of Speech, Language Pathology and Audiology, Chung Shan Medical University, Taichung, Taiwan \\ ${ }^{5}$ Department of Psychiatry, Cathay General Hospital, Taipei, Taiwan \\ ${ }^{6}$ School of Medicine, Fu Jen Catholic University, Taipei, Taiwan \\ ${ }^{7}$ Mood Disorders Psychopharmacology Unit, University Health Network, University of Toronto, Toronto, ON, USA \\ ${ }^{8}$ Department of Psychiatry, University of Toronto, Toronto, ON, USA \\ * Dr. Wei-Che Chiu contributes equally to Dr. Hsiang-Lin Chan \\ Correspondence to: Hsiang-Lin Chan, email: 8802038@adm.cgmh.org.tw
}

Keywords: hepatocellular carcinoma, antidepressants, Taiwan national insurance

Received: July 18, $2016 \quad$ Accepted: October 19, $2016 \quad$ Published: October 23, 2016

Copyright: Chen et al. This is an open-access article distributed under the terms of the Creative Commons Attribution License (CC-BY), which permits unrestricted use, distribution, and reproduction in any medium, provided the original author and source are credited.

\section{ABSTRACT}

Hepatocellular carcinoma (HCC) is highly prevalent in Asia. Antidepressants have been associated with increase in hepatocellular carcinoma. This is the first Asian population-based study to evaluate the association between antidepressant use and risk of HCC. Based on Taiwan's National Health Insurance Research Database, we conducted a nationwide population-based study. A total of 49,998 cases with HCC were identified and paired with 244,236 randomly selected controls. The data was analyzed via the conditional logistic regression model adjusting for several confounding factors. Use of tricyclic antidepressants (TCAs) and selective serotonin reuptake inhibitors (SSRIs) was associated with lower risk for HCC. No apparent association was found between use of other classes of antidepressants and HCC, including monoamine oxidase inhibitors (MAOIs), serotonin norepinephrine reuptake inhibitors (SNRIs), trazodone, mirtazapine and bupropion. The findings of a protective effect of TCAs and SSRIs for HCC should be interpreted with caution and warrants further research.

\section{INTRODUCTION}

Hepatocellular carcinoma (HCC) is the second leading cause of cancer death worldwide including both high as well as low/middle income countries [1]. HCC is a fatal cancer and the 5-year survival rate for $\mathrm{HCC}$ is $25 \%$ $40 \%$ subsequent to diagnosis [2].

Antidepressant use is common and is increasing markedly in the past 2-3 decades [3,4]. Antidepressant offer symptomatic relief for disparate disorders, including anxiety disorders, depressive disorders, sleep disorders and pain disorders [5]. It is estimated that the prevalence of long-term antidepressant use (operationalized as longer than 24 months) has doubled in the US (3.0\% to 6.9\%) from 1999 to 2010 [6]. The high level of exposure of the general population to antidepressants invites the need for careful analysis of long-term safety.

Based on their mechanism of action, antidepressants can be divided into several classes including, but not limited to, the selective serotonin reuptake inhibitors (SSRIs), serotonin-norepinephrine reuptake inhibitors (SNRIs), tricyclic antidepressants (TCAs), and 
monoamine oxidase inhibitors (MAOIs). Most classes of antidepressants are biotransformed in the liver by isoenzymes of the cytochrome $\mathrm{P} 450$ system. In a review on antidepressant-induced liver injury, Voican el al. [7] concluded that all classes of antidepressants were associated with a risk of hepatotoxicity to varying degrees. Iproniazid, nefazodone, phenelzine, imipramine, amitriptyline, duloxetine, bupropion, trazodone, tianeptine, and agomelatine are most frequently reported to induce liver injury, while citalopram, escitalopram, paroxetine, and fluvoxamine are associated with a lower risk of hepatotoxicity [7]. Drug induced liver injury is common and is one of the most common reasons drugs are terminated during development and/or removed from the market after approval [8].

Furthermore, antidepressants have long been reported to be associated with the risk of developing several cancers, including HCC. For example, one clinical study indicated a transient but significant positive association between amitriptyline (a TCA) and HCC [9]. In another study, in vitro administration of paroxetine did not indicate genotoxic effects, but administration in male mice increased the incidence of tumor malignancy [10]. Moreover, animal studies assessing the carcinogenicity of sertraline (an SSRI) found a slight increase in benign liver tumor incidence in male mice [11]. A systematic review of the US Food and Drug Administration-approved registration data reported that duloxetine (SNRIs) was associated with increased incidence of HCC in female mice, and mirtazapine (NaSSA) was associated with increased incidence of HCC in male mice [12]. However, results have been inconsistent. One study reported that amitriptyline had an anti-cancer effect, promoting enhanced oxidative damage to cancer cells, including human hepatoma cells [13]. Two more studies also showed that fluoxetine and sertraline, both SSRIs, would induce apoptosis in human hepatoma cells [14,15]. Additionally, one nationwide database record linkage study in Finland revealed that antidepressant use had no association with the incidence of liver cancer [16]. Lastly, Bendele et al. [17] administered fluoxetine to rats and mice and did not find an increase in the incidence of HCC.

The highest incidence rates of $\mathrm{HCC}$ in the world are reported in Asia and Africa [18]. In Taiwan, HCC has a particularly high incidence rate $(32.97 / 100,000)$ and the major cause is chronic HBV infection, highlighting the importance of elucidating risk factors for its development [19]. Given the concurrent high rate (4.63\%) of antidepressant use in Taiwan [20] and the extant conflicting evidence regarding an association with HCC development, we conducted a population-based nested case-control study to explore the associations between the use of antidepressants and HCC.

\section{MATERIALS AND METHODS}

The National Health Insurance (NHI) program, the current health system in Taiwan, has been in effect since March 1, 1995. It is a compulsory social insurance paid by all residents and, as of December 2008, covered 99.5\% of population [21]. The program's database, National Health Insurance Research Database (NHIRD), consists of registration files and original claim data for patient reimbursement. The data used in this study was obtained from NHIRD between January 1, 1997 and December 31, 2008, which provided detailed medical information about outpatient visits, hospital admission, prescription name and dose, medical procedures performed, and diagnostic codes.

To assess the association between antidepressant use and the incidence of $\mathrm{HCC}$, we carried out a nationwide population-based nested case-control study. The outcome of the study was occurrence of HCC. We defined cases with $\mathrm{HCC}$ as two or more outpatient diagnoses or one inpatient diagnosis of $\mathrm{HCC}$, and extracted diagnostic code information from NHI files according to the International Classification of Diseases, 9th Revision, Clinical Modification (ICD-9-CM). Each case with HCC diagnosis was linked to the Catastrophic Illness Claim Dataset to verify the diagnostic status again. The date of $\mathrm{HCC}$ claim was defined as the index date.

Five controls without a cancer diagnosis before the index date were randomly selected using incidence density sampling. The sampled date was the time of cancer cases diagnosed as HCC, defined as the index date. The controls were matched to cancer cases by year of birth, and individuals who were dead or discontinued the health insurance were excluded.

The pharmacological coding system we used to categorize antidepressants (N06A) is based on the Anatomical Therapeutic Chemical classification system [22]. We extracted prescription names and dose from the NHI database. All antidepressant use was identified and classified according to mechanism of action. This divided antidepressants into seven classes, including TCAs (i.e. amitriptyline, clomipramine, dothiepin, doxepin, imipramine, maprotiline, and melitracen), MAOIs (i.e. moclobemide, clorgyline, tranylcypromine, isocarboxacid, phenelzine, and selegiline), SSRIs (i.e. citalopram, escitalopram, fluoxetine, fluvoxamine, paroxetine, and sertraline), SNRIs (i.e., duloxetine, venlafaxine), serotonin antagonist and reuptake inhibitor (SARI) (i.e. trazodone), noradrenergic and specific serotonergic antidepressant (NaSSA) (i.e. mirtazapine), and norepinephrine dopamine reuptake inhibitor (NDRI) (i.e. bupropion).

Antidepressant exposure was standardized and the defined daily dose (DDD) according to WHO [22] was calculated. Cancer cases and controls with antidepressant use were categorized into four subgroups according to the DDDs (e.g. 28-83 DDD, 84-167 DDD, 168-335 DDD, 
Table 1: Demographic data of cases and controls

\begin{tabular}{|l|l|l|l|l|l|l|}
\hline & & \multicolumn{2}{|c|}{ Cases } & \multicolumn{2}{c|}{ Controls } & \multirow{2}{*}{$\boldsymbol{p}$ value } \\
\hline & & \multicolumn{2}{|c|}{$(\boldsymbol{n}=\mathbf{4 9 9 9 8 )}$} & \multicolumn{2}{c|}{$(\boldsymbol{n}=\mathbf{2 4 4 2 3 6})$} & \multirow{2}{*}{0.66} \\
\hline & & & & & & \\
\hline & $\geqq 40$ & 9282 & $18.56(\%)$ & 45302 & $18.55(\%)$ & \\
\hline & $41-50$ & 14028 & $28.06(\%)$ & 68910 & $28.21(\%)$ & \\
\hline & $51-60$ & 11428 & $22.86(\%)$ & 56210 & $23.01(\%)$ & \\
\hline & $61-70$ & 9650 & $19.30(\%)$ & 47011 & $19.25(\%)$ & \\
\hline & $71-80$ & 4849 & $9.70(\%)$ & 23213 & $9.50(\%)$ & \\
\hline & $\geqq 80$ & 761 & $1.52(\%)$ & 3590 & $1.47(\%)$ & \\
\hline Sex, Female & & 16258 & $32.52(\%)$ & 79615 & $32.60(\%)$ & 0.73 \\
\hline & & & & & & $<.0001$ \\
\hline & 0 & 7094 & $14.19(\%)$ & 35955 & $14.72(\%)$ & \\
\hline & $1-25000$ & 7136 & $14.27(\%)$ & 37931 & $15.53(\%)$ & \\
\hline & $25001-40000$ & 25815 & $51.63(\%)$ & 107743 & $44.11(\%)$ & \\
\hline & $\geqq 40001$ & 9953 & $19.91(\%)$ & 62607 & $25.63(\%)$ & \\
\hline & & & & & & $<.0001$ \\
\hline & Very high & 12827 & $25.66(\%)$ & 71539 & $29.29(\%)$ & \\
\hline & High & 23135 & $46.27(\%)$ & 113407 & $46.43(\%)$ & \\
\hline & Moderate & 9508 & $19.02(\%)$ & 40193 & $16.46(\%)$ & \\
\hline & Low & 4528 & $9.06(\%)$ & 19097 & $7.82(\%)$ & \\
\hline
\end{tabular}

${ }^{a}$ 1US $\$=32.3$ New Taiwan Dollars (NTD) in year 2008

${ }^{\mathrm{b}}$ Quartiles by human development index

and $\geqq 336$ DDD). Antidepressant use restricted to the year before cancer diagnosis was not included due to a potential protopathic effect [23].

Demographic variables including age at index date, income, and residential area were available in the NHI files. We performed a systematic review of the literature to identify factors that could confound our analyses, such as comorbid medical disorders and concomitant medication use. Comorbid medical disorders included depressive disorders, $\mathrm{HBV}$ infection, $\mathrm{HCV}$ infection, type 2 diabetes mellitus (DM), liver cirrhosis, and alcohol related disorders. Since NHI files didn't include smoking habit, we used chronic obstructive pulmonary disease (COPD) as proxy for smoking status. Concomitant medication use, including anti-HBV and anti-HCV medication, aspirin, non-aspirin NSAIDs, and statins, was controlled for.

\section{Statistical analysis}

All statistical analyses were conducted using SAS version 9.2. [24] Descriptive statistics were performed to compare demographic features, comorbid disorders and concomitant medication use between HCC cases and controls.

We used conditional logistic regression models to explore our main interest, the effect of antidepressant on the risk of HCC. In order to clarify the impact of antidepressants, we surveyed different classes of antidepressants and different cumulative dose of antidepressants. A total of seven classes of antidepressants were included, and we divided the cumulative exposure into four dosage ranges, as mentioned above. The crude odds ratio (OR) and the adjusted OR were calculated, in each class of antidepressants and cumulative doses. P for trend was also calculated to clarify dose-dependent effects.

To compare the percentage of antidepressant use between cancer cases and controls, crude odds ratios were calculated. The corrected odds ratios were calculated with a $95 \%$ confidence interval (CI) after adjustment for demographic features and confounding factors including depressive disorders, HBV infection, HCV infection, type $2 \mathrm{DM}$, liver cirrhosis, alcohol related disorders, COPD, anti-HBV and anti-HCV medication, aspirin, non-aspirin NSAIDs, and statins. A $p$ value of less than 0.05 was considered statistically significant.

\section{Ethics statement}

The study was approved by the Institutional Review Board of Chiayi Chang Gung Memorial Hospital.

\section{RESULTS}

We identified 49,998 HCC cases and 244,236 controls in NHI files between 1997 and 2008. The mean age of diagnosis of HCC was $57.41 \pm 13.36$ years old, while 
Table 2: Medical diseases and drugs used with cases and controls

\begin{tabular}{|c|c|c|c|c|c|}
\hline \multirow[b]{2}{*}{ Medical diseases } & \multicolumn{2}{|c|}{ Cases } & \multicolumn{2}{|c|}{ Controls } & \multirow[t]{2}{*}{$p$ value } \\
\hline & & & & & \\
\hline Depressive disorders $^{\mathrm{a}}$ & 2077 & $4.15(\%)$ & 7326 & $3.00(\%)$ & $<.0001$ \\
\hline HBV infection ${ }^{b}$ & 6502 & $13.00(\%)$ & 3883 & $1.59(\%)$ & $<.0001$ \\
\hline $\mathrm{HCV}$ infection $^{\mathrm{c}}$ & 6442 & $12.88(\%)$ & 2789 & $1.14(\%)$ & $<.0001$ \\
\hline Type 2 DM & 11042 & $22.08(\%)$ & 28262 & $11.57(\%)$ & $<.0001$ \\
\hline Liver cirrhosis $^{\mathrm{d}}$ & 25134 & $50.27(\%)$ & 23396 & $9.58(\%)$ & $<.0001$ \\
\hline Alcohol related disorders ${ }^{\mathrm{e}}$ & 1105 & $2.21(\%)$ & 945 & $0.39(\%)$ & $<.0001$ \\
\hline COPD & 7088 & $14.18(\%)$ & 27282 & $11.17(\%)$ & $<.0001$ \\
\hline \multicolumn{6}{|l|}{ Medications } \\
\hline Anti-HBV,HCV & 569 & $1.14(\%)$ & 171 & $0.07(\%)$ & $<.0001$ \\
\hline Aspirin & 6950 & $13.90(\%)$ & 33882 & $13.87(\%)$ & 0.87 \\
\hline Nonaspirin NSAIDs & 30014 & $60.03(\%)$ & 138062 & $56.53(\%)$ & $<.0001$ \\
\hline Statins & 1858 & $3.72(\%)$ & 15451 & $6.33(\%)$ & $<.0001$ \\
\hline
\end{tabular}

$\mathrm{COPD}=$ chronic obstructive pulmonary disease (ICD-9: 490*-492*), Type 2 DM=type 2 diabetic mellitus (ICD-9: 249*, 250*, A181)

${ }^{\text {a }}$ Depressive disorder (ICD-9: 296.2, 296.3, 300.4, 311).

${ }^{\mathrm{b}} \mathrm{HBV}$ infection (ICD-9: 070.2, 070.3, V02.61)

${ }^{\mathrm{c}} \mathrm{HCV}$ infection (ICD-9: 070.7, 070.41, 070.44, 070.51, 070.54, V02.62)

${ }^{\mathrm{d}}$ Liver cirrhosis (ICD-9: 571.2, 571.5, 571.6, 572.2, 572.3, 572.4, 572.8, or 573.0).

e Alcohol related disorders (ICD-9: 291, 303.0, 303.9, 305.0, 571.0, 571.1, 571.2, or 571.3)

the mean age of the controls was $57.29 \pm 13.27$ years old. The age and sex of the controls were matched to cancer cases, so there was no significant difference between the two groups. Demographic features including age, income and urbanization are listed in Table 1 . The levels of income and urbanization showed significant difference between cancer cases and controls $(p<0.001)$.

A comparison of the prevalence of comorbid medical disorders (e.g. depressive disorders, HBV infection, $\mathrm{HCV}$ infection, type $2 \mathrm{DM}$, liver cirrhosis, alcohol related disorders, and COPD) and concomitant medication use (e.g. anti-HBV and anti-HCV medication, aspirin, non-aspirin NSAIDs, and statins) are listed in Table 2 .

The overarching aim of our study was to compare the percentage of antidepressant use in cancer cases and controls. The results of the unadjusted conditional logistic regression; the stepwise conditional logistic regression after adjusting for demographic features, comorbid medical disorders, and concomitant medications use; and the $P$ for trend are listed in Table 3. After adjustment for confounding factors, the use of TCAs (28-83 DDD [adjusted OR: 0.85, 95\% CI: 0.78-0.93]; 84-167 DDD [adjusted OR: 0.80, 95\% CI: 0.69-0.92]; 168-335 DDD [adjusted OR: $0.82,95 \%$ CI: $0.69-0.98$ ]; $\geqq 336 \mathrm{DDD}$ [adjusted OR: 0.77, 95\% CI: 0.62-0.96]) and SSRIs (2883 DDD [adjusted OR: 0.82, 95\% CI: 0.73-0.92]; 84-167 DDD [adjusted OR: $0.81,95 \%$ CI: 0.69-0.97]; 168-335 DDD [adjusted OR: $0.77,95 \%$ CI: 0.64-0.93]; $\geqq 336 D D D$ [adjusted OR: $0.61,95 \%$ CI: 0.52-0.73]) were associated with lower risks for $\mathrm{HCC}$ than the control groups. The differences existed across 4 different cumulative dosages with dose-dependent effects. Among the remaining groups of antidepressants (e.g. MAOIs, SNRIs, SARI, NaSSA, NDRI), the risks for HCC had no significant difference with the control groups, except the subgroup with 84-167 DDD of SARI (adjusted OR: 0.76, 95\% CI: 0.60-0.95).

\section{DISCUSSION}

To our best knowledge, this is the first Asian population-based study to evaluate the association between antidepressant use and risk of HCC development in the general population. After adjustment for confounding factors including comorbid medical disorders and concomitant medication, TCA and SSRI prescription was associated with lower risk for HCC development with dose-dependent effects. Use of other classes of antidepressants had no association with HCC development.

Previous studies based on human and animal data had suggested the potential hepatotoxic effects of antidepressants; however, our study did not show an increased risk for HCC across mechanistically dissimilar antidepressant exposures. Our finding was more similar with the nationwide database study in Finland which revealed that antidepressant use had no association with the risk of liver cancer [16]. In that study, antidepressants including SSRIs and non-SSRIs were assessed, though non-SSRIs were not classified into subgroups, and the 
Table 3: Associations of antidepressants use and hepatocellular cancer

\begin{tabular}{|c|c|c|c|c|c|c|c|c|}
\hline \multirow{3}{*}{ Antidepressants } & \multirow{2}{*}{\multicolumn{2}{|c|}{$\begin{array}{c}\text { Cases } \\
(n=49998)\end{array}$}} & \multirow{2}{*}{\multicolumn{2}{|c|}{$\begin{array}{c}\text { Controls } \\
(n=244236)\end{array}$}} & \multicolumn{4}{|c|}{ OR } \\
\hline & & & & & \multirow{2}{*}{$\begin{array}{c}\text { Crude } \\
\left(95 \% \mathrm{CI}^{\mathrm{a}}\right)\end{array}$} & \multirow{2}{*}{$P$ for trend } & \multirow{2}{*}{$\begin{array}{l}\text { Adjusted }^{b} \\
(95 \% \text { CI) }\end{array}$} & \multirow{2}{*}{$P$ for trend } \\
\hline & $N$ & $\%$ & $\mathbf{N}$ & $\%$ & & & & \\
\hline TCAs ${ }^{\mathrm{c}}$ & & & & & & $<.0001$ & & $<.0001$ \\
\hline 28-83DDD & 1008 & 2.02 & 3740 & 1.53 & $1.32(1.23-1.41)$ & & $0.85(0.78-0.93)$ & \\
\hline 84-167DDD & 368 & 0.74 & 1505 & 0.62 & $1.19(1.06-1.34)$ & & $0.80(0.69-0.92)$ & \\
\hline 168-335DDD & 245 & 0.49 & 961 & 0.39 & $1.23(1.07-1.42)$ & & $0.82(0.69-0.98)$ & \\
\hline$\geqq 336 \mathrm{DDD}$ & 157 & 0.31 & 706 & 0.2 & $1.08(0.91-1.29)$ & & $0.77(0.62-0.96)$ & \\
\hline SSRIs $^{\mathrm{e}}$ & & & & & & $<.0001$ & & $<.0001$ \\
\hline 28-83DDD & 618 & 1.24 & 2249 & 0.92 & $1.34(1.23-1.47)$ & & $0.82(0.73-0.92)$ & \\
\hline 84-167DDD & 288 & 0.58 & 1033 & 0.42 & $1.36(1.20-1.55)$ & & $0.81(0.69-0.97)$ & \\
\hline 168-335DDD & 248 & 0.50 & 890 & 0.36 & $1.35(1.17-1.56)$ & & $0.77(0.64-0.93)$ & \\
\hline$\geqq 336 \mathrm{DDD}$ & 317 & 0.63 & 1401 & 0.57 & $1.10(0.97-1.24)$ & & $0.61(0.52-0.73)$ & \\
\hline MAOIs $^{\mathrm{d}}$ & & & & & & $<.0001$ & & 0.45 \\
\hline 28-83DDD & 797 & 1.59 & 3279 & 1.34 & $1.19(1.10-1.28)$ & & $0.93(0.85-1.03)$ & \\
\hline 84-167DDD & 404 & 0.81 & 1590 & 0.65 & $1.24(1.11-1.38)$ & & $0.92(0.81-1.05)$ & \\
\hline 168-335DDD & 446 & 0.89 & 1672 & 0.68 & $1.30(1.17-1.44)$ & & $0.95(0.83-1.08)$ & \\
\hline$\geqq 336 \mathrm{DDD}$ & 255 & 0.51 & 919 & 0.38 & $1.36(1.18-1.56)$ & & $1.09(0.92-1.29)$ & \\
\hline SNRIs $^{f}$ & & & & & & 0.0001 & & 0.49 \\
\hline 28-83DDD & 45 & 0.09 & 150 & 0.06 & $1.44(1.03-2.00)$ & & $0.78(0.49-1.22)$ & \\
\hline 84-167DDD & 33 & 0.07 & 98 & 0.04 & $1.63(1.10-2.42)$ & & $0.97(0.57-1.63)$ & \\
\hline 168-335DDD & 33 & 0.07 & 93 & 0.04 & $1.70(1.15-2.54)$ & & $0.88(0.53-1.48)$ & \\
\hline$\geqq 336 \mathrm{DDD}$ & 39 & 0.08 & 137 & 0.06 & $1.37(0.96-1.96)$ & & $0.94(0.59-1.50)$ & \\
\hline SARI $^{\mathrm{g}}$ (Trazodone) & & & & & & $<.0001$ & & 0.006 \\
\hline 28-83DDD & 471 & 0.94 & 1362 & 0.56 & $1.69(1.52-1.87)$ & & $0.99(0.87-1.14)$ & \\
\hline 84-167DDD & 158 & 0.32 & 592 & 0.24 & $1.31(1.10-1.56)$ & & $0.76(0.60-0.95)$ & \\
\hline 168-335DDD & 100 & 0.20 & 326 & 0.13 & $1.48(1.18-1.86)$ & & $0.79(0.60-1.06)$ & \\
\hline$\geqq 336 \mathrm{DDD}$ & 59 & 0.12 & 167 & 0.07 & $1.70(1.27-2.29$ & & $0.77(0.51-1.15)$ & \\
\hline $\operatorname{NaSSA}^{\mathrm{h}}$ (Mirtazapine) & & & & & & 0.036 & & 0.03 \\
\hline 28-83DDD & 22 & 0.04 & 87 & 0.04 & $1.17(0.73-1.87)$ & & $0.58(0.32-1.06)$ & \\
\hline 84-167DDD & 14 & 0.03 & 35 & 0.01 & $1.93(1.04-3.58)$ & & $0.54(0.23-1.25)$ & \\
\hline 168-335DDD & 7 & 0.01 & 30 & 0.01 & $1.13(0.50-2.57)$ & & $0.65(0.22-1.94)$ & \\
\hline$\geqq 336 \mathrm{DDD}$ & 9 & 0.02 & 25 & 0.01 & $1.66(0.77-3.56)$ & & $0.61(0.25-1.54)$ & \\
\hline NDRI'(Bupropion) & & & & & & 0.023 & & 0.78 \\
\hline 28-83DDD & 15 & 0.03 & 36 & 0.01 & $1.98(1.09-3.63)$ & & $0.97(0.43-2.23)$ & \\
\hline 84-167DDD & 4 & 0.01 & 13 & 0.01 & $1.38(0.45-4.25)$ & & $2.02(0.40-10.2)$ & \\
\hline 168-335DDD & 7 & 0.01 & 12 & 0.00 & $2.71(1.06-6.88)$ & & $0.78(0.23-2.69)$ & \\
\hline$\geqq 336 \mathrm{DDD}$ & 1 & 0.00 & 6 & 0.00 & $0.78(0.09-6.48)$ & & $0.49(0.03-7.64)$ & \\
\hline
\end{tabular}

a $95 \%$ confidence interval

${ }^{\mathrm{b}}$ Adjusting with age, urbanization, income, depressive disorders, HBV infection, HCV infection, type 2 DM, liver cirrhosis, alcohol related disorders, COPD, aspirin, NSAIDs, statins

c TCAs: tricyclic antidepressants

d MAOIs: monoamine oxidase inhibitors

e SSRIs: selective serotonin reuptake inhibitors

${ }^{f}$ SNRIs: serotonin norepinephrine reuptake inhibitors

g SARI: serotonin antagonist and reuptake inhibitor

${ }^{\mathrm{h}} \mathrm{NaSSA}$ : noradrenergic and specific serotonergic antidepressant

${ }^{i}$ NDRI: norepinephrine dopamine reuptake inhibitor 
number of liver cancer cases was much smaller $(n=415)$ than in our study [16].

Another finding in our study is that TCA and SSRI use was associated with lower risks for HCC. A lower risk of HCC associated with exposure to TCA and SSRI has not been previously reported in an Asian population. The magnitude of the lower risk identified herein requires replication and cautious interpretation. Our finding regarding lower risk with SSRIs replicates and extends evidence with SSRIs including fluoxetine and sertraline $[14,15]$. Putative mechanistic pathways are suggested by Mun et al. [14] who reported that fluoxetine induced apoptosis of a HCC line via increased oxidative stress and reduction of mitochondrial membrane potential, while Chen et al. [15] reported that sertraline-induced apoptosis of HCC line is mediated via mitogen-activated protein kinase pathway.

According to a study conducted by Amerio et al., [12] preclinical data of antidepressant use including TCAs, SSRIs, SNRIs, trazodone, mirtazapine, and bupropion were reviewed. Non-SSRI antidepressants (i.e. duloxetine) and mirtazapine were suggested to be associated with HCC in an animal study. We also included non-SSRI antidepressants in our study; however, neither SNRIs nor mirtazapine were found to be associated with increased risk of HCC. These findings provided additional information regarding the safety profiles of non-SSRI antidepressants.

Previous epidemiological studies also explored the association between antidepressant use and cancer risk of other digestive system organs [16,25,26,27,28,29]. One population-based study in the U.S. reported tricyclic antidepressant exposure was associated with increased trend of risk of esophageal adenocarcinoma [25]. No apparent association was found between antidepressant exposure and gastric cancer $[16,25,26]$. The aforementioned Finland study showed that antidepressant exposure had no association with pancreatic cancer [16]. $\mathrm{Xu}$ et al. [27] and Coogan et al. [28] both reported that SSRI exposure was associated with decreased risk for colorectal cancer. Studies on association between TCA exposure risk of colorectal cancer showed mixed and contradictory results. One study showed TCAs use had no association with the incidence of colorectal cancer [28], while the other study showed TCA use reduced colorectal cancer risk [29].

\section{Limitations and strengths}

There are several methodological limitations that affect inferences and interpretations that can be made from our data. For example, we cannot assure accuracy in pathological biopsy diagnosis nor can there be assurances that recorded antidepressant prescriptions and their respective doses were adhered to. Moreover, there is no mechanism to determine whether antidepressants prescription is commensurate with antidepressant utilization. Moreover, the NHI files did not include records of other potential confounding factors including smoking and lifestyles which may be also related to the occurrence of HCC.

Notwithstanding the limitations, several strengths are inherent in this study. As the data were extracted from a nationwide database, the sample size was large and selection bias is minimized. The principal strength of this analysis is the perspective offered by the large database representative of the Taiwanese population.

\section{CONCLUSIONS}

In conclusion, most classes of antidepressant use had a null association with the incidence of HCC. Our findings regarding the slightly protective effect of TCAs and SSRIs use should be interpreted cautiously, and warrant further investigation

\section{ACKNOWLEDGMENTS}

The authors would like to thank Center of Excellence for Chang Gung Research Datalink (CORPG6D0161) for the comments and assistance in data analysis. This study was based on the National Health Insurance Research Database provided by the Central Bureau of National Health Insurance, the Department of Health, and managed by the National Health Research Institutes. The interpretation and conclusions contained herein do not represent those of Bureau of National Health Insurance, Department of Health, or National Health Research Institutes.

\section{CONFLICTS OF INTEREST}

The authors declare no competing financial interests.

\section{FUNDING}

The present study is supported in part by the Chang Gung Medical Foundation, Chiayi Chang Gung Memorial Hospital (CMRPG6E0271). The funders had no role in study design, data collection and analysis.

\section{REFERENCES}

1. Torre LA, Bray F, Siegel RL, Ferlay J, Lortet-Tieulent J and Jemal A. Global cancer statistics, 2012. CA: a cancer journal for clinicians. 2015; 65:87-108.

2. Kim Y, Ejaz A, Tayal A, Spolverato G, Bridges JF, Anders RA and Pawlik TM. Temporal trends in population-based death rates associated with chronic liver disease and liver 
cancer in the United States over the last 30 years. Cancer. 2014; 120:3058-3065.

3. Hemels ME, Koren G and Einarson TR. Increased use of antidepressants in Canada: 1981-2000. The Annals of pharmacotherapy. 2002; 36:1375-1379.

4. Olfson $M$ and Marcus SC. National patterns in antidepressant medication treatment. Archives of general psychiatry. 2009; 66:848-856.

5. Noordam R, Aarts N, Verhamme KM, Sturkenboom MC, Stricker BH and Visser LE. Prescription and indication trends of antidepressant drugs in the Netherlands between 1996 and 2012: a dynamic population-based study. European journal of clinical pharmacology. 2015; 71:369375.

6. Mojtabai R and Olfson M. National trends in long-term use of antidepressant medications: results from the U.S. National Health and Nutrition Examination Survey. The Journal of clinical psychiatry. 2014; 75:169-177.

7. Voican CS, Corruble E, Naveau S and Perlemuter G. Antidepressant-induced liver injury: a review for clinicians. The American journal of psychiatry. 2014; 171:404-415.

8. McIntyre RS, Panjwani ZD, Nguyen HT, Woldeyohannes HO, Alsuwaidan M, Soczynska JK, Lourenco MT, Konarski JZ and Kennedy SH. The hepatic safety profile of duloxetine: a review. Expert opinion on drug metabolism \& toxicology. 2008; 4:281-285.

9. Selby JV, Friedman GD and Fireman BH. Screening prescription drugs for possible carcinogenicity: eleven to fifteen years of follow-up. Cancer research. 1989; 49:57365747.

10. Kelvin AS, Mitchell ID and White DJ. General and genetic toxicology of paroxetine. Acta psychiatrica Scandinavica Supplementum. 1989; 350:34-36.

11. Davies TS and Kluwe WM. Preclinical toxicological evaluation of sertraline hydrochloride. Drug and chemical toxicology. 1998; 21:521-537.

12. Amerio A, Galvez JF, Odone A, Dalley SA and Ghaemi SN. Carcinogenicity of psychotropic drugs: A systematic review of US Food and Drug Administration-required preclinical in vivo studies. The Australian and New Zealand journal of psychiatry. 2015.

13. Cordero MD, Sanchez-Alcazar JA, Bautista-Ferrufino MR, Carmona-Lopez MI, Illanes M, Rios MJ, GarridoMaraver J, Alcudia A, Navas P and de Miguel M. Acute oxidant damage promoted on cancer cells by amitriptyline in comparison with some common chemotherapeutic drugs. Anti-cancer drugs. 2010; 21:932-944.

14. Mun AR, Lee SJ, Kim GB, Kang HS, Kim JS and Kim SJ. Fluoxetine-induced apoptosis in hepatocellular carcinoma cells. Anticancer research. 2013; 33:3691-3697.

15. Chen S, Xuan J, Wan L, Lin H, Couch L, Mei N, Dobrovolsky VN and Guo L. Sertraline, an antidepressant, induces apoptosis in hepatic cells through the mitogenactivated protein kinase pathway. Toxicological sciences. 2014; 137:404-415.
16. Haukka J, Sankila R, Klaukka T, Lonnqvist J, Niskanen L, Tanskanen A, Wahlbeck K and Tiihonen J. Incidence of cancer and antidepressant medication: record linkage study. International journal of cancer. 2010; 126:285-296.

17. Bendele RA, Adams ER, Hoffman WP, Gries CL and Morton DM. Carcinogenicity studies of fluoxetine hydrochloride in rats and mice. Cancer research. 1992; 52:6931-6935.

18. McGlynn KA and London WT. The global epidemiology of hepatocellular carcinoma: present and future. Clinics in liver disease. 2011; 15:223-243, vii-X.

19. Hung GY, Horng JL, Yen HJ, Lee CY and Lin LY. Changing incidence patterns of hepatocellular carcinoma among age groups in Taiwan. Journal of hepatology. 2015; 63:1390-1396.

20. Wu CS, Shau WY, Chan HY, Lee YC, Lai YJ and Lai MS. Utilization of antidepressants in Taiwan: a nationwide population-based survey from 2000 to 2009 . Pharmacoepidemiology and drug safety. 2012; 21:980-988.

21. National Health Insurance Administration, Ministry of Health and Welfare. Overview of National Health Insurance. Taipei: National Health Insurance Administration, Ministry of Health and Welfare. 2013 [in Chinese].

22. World Health Organization Collaborating Centre for Drug Statistics Methodology. ATC classification index with DDDs. WHO. (2013) Available at: http://www.whocc.no/ atc_ddd_publications/atc_ddd_index/. (Accessed 10 July 2014)

23. Rothman KJ. Induction and latent periods. American journal of epidemiology. 1981; 114:253-259.

24. SAS Institute: The SAS system for Windows. Release 9.2. . SAS Inst.., Cary, NC. 2011.

25. Vaughan TL, Farrow DC, Hansten PD, Chow WH, Gammon MD, Risch HA, Stanford JL, Schoenberg JB, Mayne ST, Rotterdam H, Dubrow R, Ahsan H, West AB, Blot WJ and Fraumeni JF, Jr. Risk of esophageal and gastric adenocarcinomas in relation to use of calcium channel blockers, asthma drugs, and other medications that promote gastroesophageal reflux. Cancer epidemiology, biomarkers \& prevention. 1998; 7:749-756.

26. Hsieh YH, Chiu WC, Lin CF, Chan HL, Liang HY, Lee Y, McIntyre RS and Chen VC. Antidepressants and Gastric Cancer: A Nationwide Population-Based Nested CaseControl Study. PloS one. 2015; 10:e0143668.

27. Xu W, Tamim H, Shapiro S, Stang MR and Collet JP. Use of antidepressants and risk of colorectal cancer: a nested case-control study. The Lancet Oncology. 2006; 7:301-308.

28. Coogan PF, Strom BL and Rosenberg L. Antidepressant use and colorectal cancer risk. Pharmacoepidemiology and drug safety. 2009; 18:1111-1114.

29. Walker AJ, Card T, Bates TE and Muir K. Tricyclic antidepressants and the incidence of certain cancers: a study using the GPRD. British journal of cancer. 2011; 104:193197. 